\title{
Functional Outcome of Humeral External Rotation Osteotomy in ERB's Palsy
}

\author{
Ahmed Shamma, Galal Mansour, Mostafa Salah Mohamed* \\ Department of Orthopedic Surgery, Faculty of Medicine, Al-Azhar University, Cairo, Egypt \\ * Corresponding author: Mostafa Salah Mohamed, E-mail: must7088@yahoo.com
}

\section{ABSTRACT}

Background: Shoulder internal rotation contracture is the most common deformity affecting the shoulder in patients with Erb's palsy. With progression of the deformity, the glenohumeral joint become more dysplastic which may lead to severe functional impairment of the affected limb.

Aim of the Work: was to evaluate the functional outcome of external humeral rotational osteotomy.

Patients and Methods: This is a prospective study on 10 patients with Erb's palsy who underwent external humeral rotational osteotomy. In this study the patients were evaluated for improvement of abduction and external rotation of shoulder after at least 6 months of operation.

Results: The mean preoperative external rotation improved from $-40^{\circ}$ to $41^{\circ}$ and the mean preoperative abduction improved from $135^{\circ}$ to $150^{\circ}$. The mean mallet score for preoperative external rotation improved from 2.20 to 4 and the mean mallet score for preoperative abduction show no difference as all patients could raise their arms above 90 degree pre and postoperatively.

Conclusion: External humeral rotational osteotomy improves shoulder function in patients with brachial plexus birth palsy suffering from internal rotation contracture associated with advanced glenohumeral joint deformity.

Keywords: Humeral External Rotation, Osteotomy in ERB's Palsy, Obstetric Brachial Plexus Palsy

\section{INTRODUCTION}

Obstetric brachial plexus palsy (OBPP) occurs due to injury of the nerve roots of the upper extremity in the neck which may occur in case of difficult delivery. Erb's palsy $(\mathrm{C} 5,6)$ is a very common type of OBPP. Most of the infants with Erb's palsy will show spontaneous recovery ${ }^{(1,2)}$. However, some of them show internal rotation contracture of the shoulder joint due to lack of opposition to muscle forces generated by latissmus dorsi, pectoralis major, subscapularis and teres major muscles.If this internal rotation deformity is not repaired, it may progress to posterior dislocation of the shoulder joint with bony deformity of the glenoid $^{(3)}$.

Surgical treatment of the internal rotation contracture is either by soft tissue release or by bony osteotomy. Traditionally, the osteotomy is done for older children with advanced glenohumeral deformity. The direction of the osteotomy is external rotation osteotomy aiming to improve the function of the upper extremity. This osteotomy ignores the glenohumeral deformity as it may lead to posterior dislocation of the humeral head in relation to the glenoid. ${ }^{4}$.

The aim of the current work was to evaluate the functional outcome of external humeral rotational osteotomy in children with Erb's palsy.

\section{PATIENTS AND METHODS}

This prospective study included a total of 10 patients with Erb's palsy who underwent external humeral rotational osteotomy attending at Al-|Azhar University Hospitals. Approval of the ethical committee and a written informed consent from all the subject's parents were obtained. This study was conducted between January 2017 and June 2018.

\section{Inclusion criteria:}

Older Children with limited active and passive external rotation and associated with advanced glenohumeral deformity.

\section{Exclusion criteria:}

Other paralytic conditions.

All patients are subjected to carful history taking and open communication with their parents and the functional deficits. Careful examination is also done.

The patient should be properly exposed to permit complete inspection of both shoulders. Swelling, asymmetry, muscle wasting, scars, ecchymosis, scapular dyskinesia, infection and any venous distention 
should be noted. Also focusing on the way the patient carries and move his limb.

Include palpating bony prominences and soft tissue around the shoulder. Bony structures should include: the acromioclaviular joint, the sternoclavicular joint, the clavicle, the borders of the scapula, and the greater, lesser tuberosities of the humerus and the coracoid process.

Because of the wide range of motion of the shoulder, the affected extremity should be compared with the unaffected side to determine the patient's normal range. Active and passive range of motion should be assessed (abduction, adduction, flexion, extension, external rotation, internal rotation).

Improvement of external rotation and abduction of the shoulder were evaluated based on mallet score system.

\section{The Modified Mallet Score System:}

The Modified Mallet Classification System categorizes the upper limb with respect to five natural actions: global abduction of the arm, touching the hand to the back, external rotation of the arm, placing the hand behind the head, and raising the hand to the mouth. Because the test actions involve shoulder and elbow movements, it predominantly reflects the integrity of the $\mathrm{C} 5$ and $\mathrm{C} 6$ roots and the upper trunk of the brachial plexus ${ }^{(5)}$.

\section{Surgical technique}

Patients are positioned in the modified beach chair position, with the affected limb prepped and draped into the field; the humerus is exposed by deltopectoral approach, centered over the level of the deltoid insertion. The plane between deltoid and pectoralis major muscle is developed then the periosteum is incised longitudinally and subperiosteal interval is developed.

After the level of osteotomy is marked, single 3.5-mm dynamic compression small plate is used. It is important to control all bony fragments so that the proximal holes are drilled first then tapped. Two k-wires may be drilled into the humerus proximal and distal to the osteotomy site in a parallel fashion to control the amount of rotation. A transverse osteotomy is then done with a small oscillating blade just proximal to the deltoid insertion. The distal fragment is externally rotated to the desired amount to allow for better functional positioning of the hand. Usually, 60 degrees to 90 degrees of external rotation is preferred, and the previously placed K-wires or humeral markings may be used to guide rotation. The plate is applied to the proximal segment and the previously drilled screws are applied. Before placement of the distal screws, intraoperative checking of the amount of rotation is done to avoid overcorrection. Care is made to ensure that hand can touch abdomen, perineum and midline easily. One the desired amount of rotation is achieved, the distal screws are placed and rigid fixation is achieved. Ideally, 4 to 6 cortices of fixation should be obtained proximal and distal to the osteotomy. The periosteum is then reapproximated over the plate. Subcutaneous tissues are closed in layers and skin is closed.

Postoperatively, patients are managed with immobilization of the upper extremity in a broad arm sling. Immobilization is typically discontinued after four to six weeks, following radiographic confirmation of osseous healing. Patients subsequently undergo physical therapy for range of motion and strengthening exercises if needed.

\section{Statistical Methods}

The collected data was revised, coded, tabulated and introduced to a PC. IBM SPSS statistics (V. 25.0, IBM Corp., USA, 20172018) for data analysis. Data were presented and suitable statistical analysis was done according to the type of data obtained for each parameter. $\mathrm{P}<0.05$ was considered significant, $p<0.01$ was considered highly significant and p> 0.05 was considered non-significant.

\section{RESULTS}

The pool of patients consisted of 6 males and 4 females. Table (1) Seven patients had right extremity involvement, whereas the remaining three patients had involvement of the left extremity \& there was no bilateral involvement. Table (2).

All patient had C5,C6 lesions, no patient had C7 involvement or complete brachial plexus palsy.

The mean age of patient at time of operation was 8 years ranged from 6 to 10 
years. They had an average postoperative follow-up period ranged from 6 months to 12 months. Table (3).

According to range of motion, table (4), the mean preoperative external rotation was $-40^{\circ}$ and postoperative external rotation was $41^{\circ}$ with mean gain $81^{\circ}$.This shows highly statistically significant improvement of external rotation with $\mathrm{p}$ value $<0.001$.

The mean preoperative abduction was $135^{\circ}$ and postoperative abduction was $150^{\circ}$ with mean gain 15 degree. This shows highly statistically significant improvement of abduction with $p$ value $<0.001$.

According to mallet score, table (5), the mean mallet score for preoperative external rotation was 2.20 and the mean mallet score for postoperative external rotation was 4 . This shows statistically highly significant improvement of mallet score for external rotation with $\mathrm{p}$ value $<0.001$.

The mean mallet score for preoperative hand to neck was 2.40 and the mean mallet score for postoperative hand to neck is 3.80 . This shows statistically highly significant improvements of mallet score for hand to head with $\mathrm{p}$ value $<0.001$.

The mean mallet score for preoperative hand to mouth was 2.30 and the mean mallet score for postoperative hand to mouth is 4.This shows statistically highly significant improvement of mallet score for hand to mouth with $\mathrm{p}$ value $<0.001$.

In all children the hand could reach mouth preoperatively but with trumpet sign (the shoulder is abducted as in blowing the trumpet) but postoperatively they can reach mouth easily in a more functional position without trumpeting.

Table (1): Distribution of the patient according to sex.

\begin{tabular}{|l|c|c|}
\hline \multicolumn{1}{|c|}{ Sex } & Number of patients & Percentage \\
\hline Male & 6 & $60 \%$ \\
\hline Female & 4 & $40 \%$ \\
\hline
\end{tabular}

Data are expressed as frequency and percentage data.

Table (2): Distribution of the patient according to side of affection

\begin{tabular}{|l|c|c|}
\hline Side affection & Number of patients & Percentage \\
\hline Right & 7 & $70 \%$ \\
\hline Left & 3 & $30 \%$ \\
\hline
\end{tabular}

Data are expressed as frequency and percentage data.

Table (3): Age

\begin{tabular}{|l|c|c|c|c|}
\hline & Min. & Max. & Mean & \pm SD \\
\hline Age at operation & 6 & 10 & 8 & 1,49 \\
\hline
\end{tabular}

Table (4): Preoperative and postoperative data of abduction and external rotation

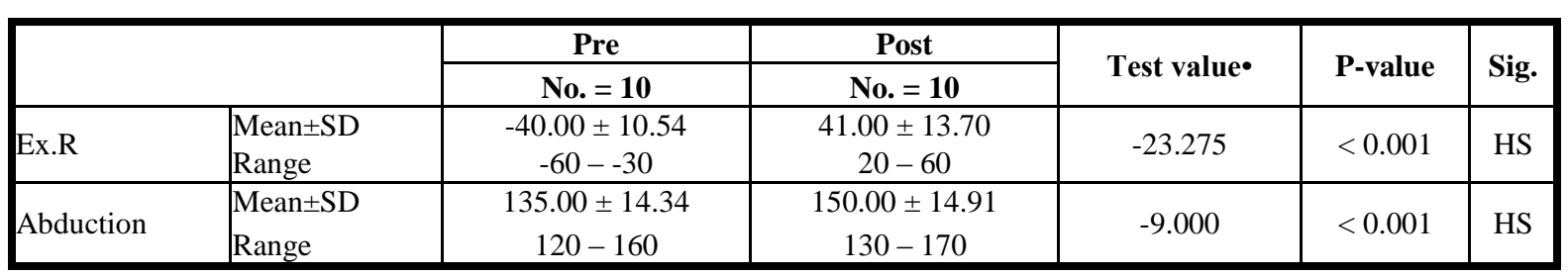

P-value $>0.05$ : Non significant (NS); P-value <0.05: Significant (S); P-value $<0.01$ : highly significant (HS)

•: Paired t- test

Table (5): Preoperative and postoperative mallet score for global function of the shoulder.

\begin{tabular}{|c|c|c|c|c|c|c|}
\hline & Pre & Post & \multirow{2}{*}{ Test value• } & \multirow{2}{*}{ P-value } & \multirow{2}{*}{ Sig. } \\
\hline & & No. $=10$ & No. $=10$ & & & \\
\hline Abduction & $\begin{array}{l}\text { Mean } \pm \text { SD } \\
\text { Range }\end{array}$ & $\begin{array}{c}4.00 \pm 0.00 \\
4-4\end{array}$ & $\begin{array}{c}4.00 \pm 0.00 \\
4-4\end{array}$ & NA & NA & NA \\
\hline Ex.R & $\begin{array}{l}\text { Mean } \pm \text { SD } \\
\text { Range }\end{array}$ & $\begin{array}{c}2.20 \pm 0.42 \\
2-3\end{array}$ & $\begin{array}{c}4.00 \pm 0.00 \\
4-4\end{array}$ & -13.500 & 0.000 & HS \\
\hline Hand to neck & \begin{tabular}{|l|} 
Mean \pm SD \\
Range
\end{tabular} & $\begin{array}{c}2.40 \pm 0.52 \\
2-3\end{array}$ & $\begin{array}{c}3.80 \pm 0.42 \\
3-4\end{array}$ & -8.573 & 0.000 & HS \\
\hline Hand to mouth & $\begin{array}{l}\text { Mean } \pm \text { SD } \\
\text { Range }\end{array}$ & $\begin{array}{c}2.30 \pm 0.48 \\
2-3\end{array}$ & $\begin{array}{c}4.00 \pm 0.00 \\
4-4\end{array}$ & -11.129 & 0.000 & HS \\
\hline
\end{tabular}

P-value $>0.05$ : Non significant (NS); P-value <0.05: Significant (S); P-value $<0.01$ : highly significant (HS)

$\bullet:$ Paired t- test 


\section{DISCUSSION}

This is a prospective study conducted on ten patients who underwent external humeral rotational osteotomy. Current study addressed the treatment of internal rotation deformity of the shoulder associated with advanced glenohumeral deformity by performing external rotational osteotomy of the proximal humerus.

The pool of patients consisted of six boys and four girls. Seven patients had right extremity involvement, whereas the remainder three patients had involvement of the left extremity $\&$ there was no bilateral involvement.

In this study ages at the time of surgery spanned from 6 years to 10 years, with an average age 8years. All patients had C5, 6 lesion, no patient had $\mathrm{C} 7$ involvement or complete brachial plexus palsy.

In this group of children with Erb's palsy in whom the main functional deficit of the limb was related to internal rotation contracture of the shoulder, preoperative radiologic assessment showed deformity of the glenohumeral joint.

In this study the duration of follow up ranged from 6 months to 12 months with mean 9.02 months.

Various techniques have been used for humerus osteotomy in Erb's palsy. Zancolli and Zancolli ${ }^{(\boldsymbol{6})}$ used a lower axillary incision, divided the distal part of pectoralis major tendon, and performed the osteotomy at the level of the distal border of the pectoralis major tendon. . Al Zahrani ${ }^{(7,8)}$ performed the osteotomy just below the deltoid insertion and removed an anterior wedge from the proximal segment of the humerus, which helped in bringing the hand into more flexion toward the face and mouth. Cuesta et al. ${ }^{(9)}$, Goddard and Fixsen $^{(10)}$ used a deltopectoral incision and performed the osteotomy just above the deltoid insertion and this technique was used in this study. Regardless of the technique of osteotomy, overcorrection should be avoided because a fixed external rotation posture is functionally more disabling than the internal rotation contracture.

In this study we evaluate the effect of the external humeral rotational osteotomy on the function of the shoulder by using values of external rotation in degrees and also according to modified mallet scoring system.

The osteotomy indirectly facilitated abduction in this study. This observation was noted by other investigators ${ }^{(8,9)}$ who believed that the osteotomy allowed the long head of the biceps to become more active as a shoulder abductor and increase the mechanical advantage of the deltoid muscle.

About the results of our study after one year follow up, the mean external rotation improved from -40 preoperatively to41 postoperatively with gain 80 in external rotation. The mean abduction improved from 135 preoperatively to 150 postoperatively with gin 15 in abduction.

According to mallet scoring the mean external rotation score improved from 2.20 preoperatively to 4 postoperatively. All patients became able to reach their hands to mouth easily in a more functional position without trumpeting. Also they could reach their necks easily.

Akinci et al. ${ }^{(11)}$ performed external rotation osteotomy of the humerus on 40 children ( 24 boys, 16 girls: mean age 7.5 years). Involvement was at c5-6 roots at 11 patients, c5-6-7 roots in 19 patients, and at all roots in 10 patients. All patients had humeral head deformity on radiological investigation. The results showed that the mean postoperative abduction improved from 80 (range from 0 to 170 degrees) to 95(range from 30 degrees to 170 degrees) with gain 15 and also internal rotation contracture improved in all patients. Passive total shoulder rotation remains unchanged following surgery.

In Al Qattan ${ }^{(12)}$, fifteen consecutive patients with a mean age of 6.5 years were included. In all children glenohumeral deformity was confirmed by preoperative radiology. The humerus osteotomy was done just below the insertion of the deltoid muscle, and fixation was done with stainless steel plate and screws. External rotation of the shoulder improved from mean $-50^{\circ}\left(-30^{\circ}\right.$ to $\left.-60^{\circ}\right)$ and to $30^{\circ}$ (range from $20^{\circ}$ to $50^{\circ}$ ) with gain 80 in external rotation. The results were assessed using modified Mallet score. Before surgery 13 children had a score of 2 and 2 children had a score of 3. At final follow-up evaluation (range, 
1-5 years; average, 3 years), all 15 children improved shoulder function and obtained a modified Mallet score of 4 and achieved slight improvement of shoulder abduction and elbow extension. All children could wash, dress, perform self-cleaning activities and feed themselves better and no longer needed help with these activities.

Waters and Bae ${ }^{(13)}$ performed a study on Forty-three patients underwent a derotational humeral osteotomy and followed for a minimum of two years (average, 3.7 years). The average age of the patients at the time of surgery was 7.6 years (range, 2.3 to 17.0 years). The average amount of external rotation gained with osteotomy was 64 degrees (range, 35 degrees to 90 degrees). The mean Mallet classification scores for the individual elements similarly demonstrated improvement following osteotomy, with the greatest gains in hand-tomouth, external rotation and hand-to-neck motions.

In this study almost near daily functional requirements of the upper limb like using hand in washing face, eating, combing hair can be reached in children with internal rotation deformity associated with glenohumeral dysplasia by reorienting the arm in a more functional position.

\section{CONCLUSION}

It could be concluded that external humeral rotational osteotomy improves shoulder function in patients with brachial plexus birth palsy and internal rotation contracture associated with severe glenohumeral deformity precluding soft tissue releases and tendon transfers.

\section{REFERENCES}

1. Greenwald AG, Schute PC, Shiveley JL (1984): Brachial plexus birth palsy: a 10year report on the incidence and prognosis. J Pediatr Orthop., 4:689-92.

2. Abdelgawad A, Naga O (2014): Birth injuries and orthopedic manifestations in newborns.

https://link.springer.com/chapter/10.1007/ 978-1-4614-7126-4_5
3. Hoeksma AF, Ter Steeg AM, Dijkstra PF, Nelissen RG, Beelen A, de Jong BA (2003): Shoulder contraction and bony deformity in obstetrical brachial plexus injury. J Bone Joint Surg., 85A:316-22.

4. Zancolli EA (1981): Classification and management of the shoulder in birth palsy. Orthop Clin North Am., 12:433-57.

5. Piatt JH (2004): Birth Injuries of the Brachial Plexus. Pediatric Clinics of North America,51 (2): 421-440.

6. Zancolli EA, Zancolli ER (1988): Palliative surgical procedures in sequelae of obstetric palsy. Hand Clin., 4:643-669.

7. Al Zahrani S (1993): Modified rotational osteotomy of the humerus for Erb's palsy. Int Orthop., 17:202-204.

8. Al-Zahrani S (1997): Combined Sever's release of the shoulder and osteotomy of the humerus for Erb's palsy. J Hand Surg., 22B:591-593.

9. Cuesta FJG, Parts FL, Lopez FJG, Sitja JB (1982): The role of bone operations as palliative surgical treatment for the sequelae of obstetrical brachial paralysis in the shoulder. Acta Orthop Belg.; 48:757761.

10. Goddard NJ, Fixsen JA (1984): Rotation osteotomy of the humerus for birth injuries of the brachial plexus. J Bone Joint Surg., 66B:257-259.

11. Akinci $M^{1}$, Ay $S$, Kamiloğlu $S$, Erçetin $O$ (2005):External rotation osteotomy of the humerus for the treatment of shoulder problems secondary to obstetric brachial plexus palsy. Acta Orthop Traumatol Turc., 39(4):328-33.

12. Al-Qattan A (2002): Rotation Osteotomy of the Humerus for Erb's Palsy in Children With Humeral Head Deformity. $J$ Hand Surg., 27A:479-483.

13. Waters PM, Bae DS (2006): The effect of derotational humeral osteotomy on global shoulder function in brachial plexus birth palsy. J Bone Joint Surg Am.,88(5):103542. 